\title{
Outcome of management of neurosurgical patients in the intensive care unit: An early clinical audit of a single institution from a developing country
}

\author{
Joseph Obande, ${ }^{1}$ Ega Otorkpa, ${ }^{1}$ Elisabeth Obande ${ }^{2}$ \\ ${ }^{1}$ Division of Neurosurgery, Department of Surgery, University of Abuja Teaching Hospital, Gwagwalada, \\ Abuja; ${ }^{2}$ School of Post-Basic Critical Care Nursing, University of Abuja Teaching Hospital, Gwagwalada, \\ Abuja, Nigeria
}

\begin{abstract}
Care of neurosurgical patients in the Intensive Care Unit (ICU) has been evolving in recent times. This included fast growing new
\end{abstract}

\begin{abstract}
Correspondence: Joseph Obande, Division of Neurosurgery, Department of Surgery, University of Abuja Teaching Hospital, Gwagwalada, Abuja, Nigeria.

E-mail: obandejo@yahoo.com

Key words: Critical care; mortality; neurocritical care; traumatic brain injury.

Contributions: JO was involved in the conceptualization and design, drafting and revising of the article. He agreed to take responsibility to be accountable to the manuscript and shall consent to the final approval. EO was involved in the interpretation of the data, revising the article and accepting to give final approval for publishing and accountable for the article. EO was involved in the analysis and statistical work, revising the article, and accepts to give final approval for publishing and accountable for the article.
\end{abstract}

Conflict of interest: The authors declare no conflict of interest.

Ethics approval and consent to participate: Though, the study is a retrospective chart review, ethical approval was sought from the Health Research Ethics Committee of the University of Abuja Teaching Hospital, Abuja, Nigeria.

Consent for publication: Not applicable.

Availability of data and materials: The data that support the findings of this study are available on request from the corresponding author, JO. The data are not publicly available due to their containing information that could compromise the privacy of research participants, who were managed at the described hospital.

Received for publication: 10 June 2020.

Revision received: 12 January 2021.

Accepted for publication: 22 January 2021.

This work is licensed under a Creative Commons Attribution NonCommercial 4.0 License (CC BY-NC 4.0).

(C) Copyright: the Author(s), 2021

Licensee PAGEPress, Italy

Annals of Clinical and Biomedical Research 2021;2:89

doi:10.4081/acbr.2021.89 techniques in neuromonitoring, neuroimaging and enhanced therapeutic tools. The pace of evolution has not been replicated in resource-constrained setting such as in Nigeria, despite overwhelming evidence of its benefit to neurological patients. Patients' outcome, including death, in the ICU is a reflection of quality of care assessed during the hospitalization process; as many neurosurgical patients require ICU bed in the course of hospital care. The objective was to characterize the profile of the patients, mortality and factors associated with mortality while in the ICU at the dawn of commencement of neurosurgical services at our centre. A descriptive retrospective study utilizing patients' datasets of all neurosurgical admissions into the ICU of a tertiary hospital, University of Abuja Teaching Hospital, Abuja, between 2013 and 2015 were analyzed for deaths and factors associated to death. During the 3-years period, 19 neurosurgical deaths occurred in the ICU of University of Abuja Teaching Hospital, translating to a mortality of $63.3 \%$. The majority of the deceased patients $(n=22$, $71 \%$ ), was of the young age group and died from head trauma. 14 $(73.7 \%)$ presented late to the hospital, and all the deceased presented late to the ICU. 17 (89.5\%) had head injury, of which, $84.2 \%$ was from severe head injury. All the deceased who were administered mannitol died. The early data suggest an unusually high mortality among neurosurgical patients managed in the ICU. A reflected application of modern intensive care measures might lead to increased survival of neurocritically ill patients.

\section{Introduction}

Globally, the need for specialized medical care is changing the face of Intensive Care units. ${ }^{1-3}$ As many neurosurgical patients require ICU during their hospital care, treatment of neurosurgical patients in a specialized neurosurgical intensive care unit has proven to be of benefit, including management and outcome. ${ }^{4-6}$ In Nigeria, this practice is less obvious. ${ }^{7}$ There has been a progressive upscaling of neurosurgical services due to availability of manpower; the patient load is increasing, and radiodiagnostic capacities are improving, but limited availability of financial resources challenges neurological intensive care growth. Tertiary health facilities need to adapt ways to overcome this challenge to ensure optimum service delivery.

The University of Abuja Teaching Hospital, Abuja, has a fourbedded level-I mixed medical-surgical Intensive Care Unit (ICU). In terms of ventilated patients in the ICU, clinical neurological evaluation such as the Glasgow Coma Scale score, is difficult, making reliance on both noninvasive and invasive monitors like electroencephalography, intracranial pressure monitors, essential. However, these are not routinely available in our environment yet. Therefore, neurological and cardiopulmonary surveillance are the 
mainstay of monitoring. ICU connotes extensive diagnostics, and artificial ventilation including the weaning process are prime reasons for often prolonged ICU stay and high cost of care. The presence of an intensivist, critical care physician or even, a neurocritical care physician is of utmost benefit. ${ }^{8}$

Neurosurgical services having only being a recent innovation at the hospital, in December 2012, neurosurgical patients started accounting for a disproportionately high bed occupancy at the ICU of the University of Abuja Teaching Hospital, Abuja, and given that serious neurological disease carries a relatively poorer outcome, an early management audit was imperative. The aim of the present study was, therefore, to characterize the profile of the patients, mortality and factors associated with mortality while in the ICU at the dawn of commencement of neurosurgical services. Thus, this study was conducted with the objective that the findings will be helpful for implementation of strategies that would help decrease mortality of neurosurgical patients in ICU and improve the practice.

\section{Materials and Methods}

In this descriptive study, we recorded and analyzed the profile of age, indications for admission, time to presentation and deaths of neurosurgical patients at the ICU of the University of Abuja Teaching Hospital, Abuja, Nigeria, between January 2013 and December 2015. The Patients were further characterized with respect to age, other concomitant injuries (polytrauma), use of mannitol, GCS, placement/ weaning of endotracheal intubation and surgical interventions. All information was obtained from patients' medical records, following approval from the HREC of the University of Abuja Teaching Hospital, Abuja.

\section{Results}

During the period surveyed, 31 neurosurgical patients were admitted into the ICU, complete records were found for 30 patients and so only the 30 patients were included in the study. Males were $26(86.7 \%)$ and females $4(13.3 \%)$ with a male:female of $6.5: 1$ (Figure 1). The median age group admitted was 21-29; and 22 (71\%) patients, were of the productive age group of 21-60 (Figure 2). The factors associated with death are seen in Table 1.
One of the patients with head injury was also polytraumatized and had associated traumatic splenic injury. This necessitated an explorative laparotomy, as seen in Table 1.

\section{Discussion}

\section{Time to presentation}

As summarized in Table 1, 6 (31.6\%) patients presented to the hospital after the 4-hours golden time ${ }^{9}$ for neurosurgical intervention for intracranial haemorrhages. A further 7 (36.8\%) patients presented between $24-48$ hours. Overall, $73.8 \%$ of those who died presented late. This may not be unconnected to the poor transportation and health referral systems, including delays at peripheral and secondary health centres, waiting for patients to begin to deteriorate before referral. With delays, secondary brain injuries worsen, and prognosis steeps.

Presentation to ICU from the emergency room or ward as the case may be presents a challenge. Often times, the prohibitive cost of ICU admission is the main constraint inhibiting the access to early neurocritical care needed to optimize these very ill patients. In a clime where patients pay out-of-pocket for healthcare services, and the initial deposit is prohibitively out of reach of the average citizenry, extra efforts are made, causing delays. The ICU admission is such that without the initial deposit you may not be admitted into the facility. This is a huge burden to optimal care. As much as $10(53.6 \%)$ patients were only able to be admitted into the ICU after a day of prescribing the need for ICU admission. All these impart on time to intervention, besides, time to craniotomy has been found to be risk factor for mortality. ${ }^{10}$ Indication for admission

Sixteen of the 19 patients who died, constituting $84.2 \%$ of the total (Table 1), were admitted for severe head injury. Severe head injured patients are by definition ICU candidates. But modern ICU is meant to provide a more promising care for this subset of patients. ${ }^{11}$ Then, ICU protocols and the much needed multidisciplinary care were yet to be established. Another key necessity for optimal care of the head injured patient being a CT was yet to be available in the hospital. Of the 19 who died only 10 had a brain CT scan. A significant midline shift on CT is associated with mortality. ${ }^{12}$ One of the patients who died, had moderate head injury with associated cervical spinal cord injury, but died from worsening respiratory distress from respiratory fatigue.

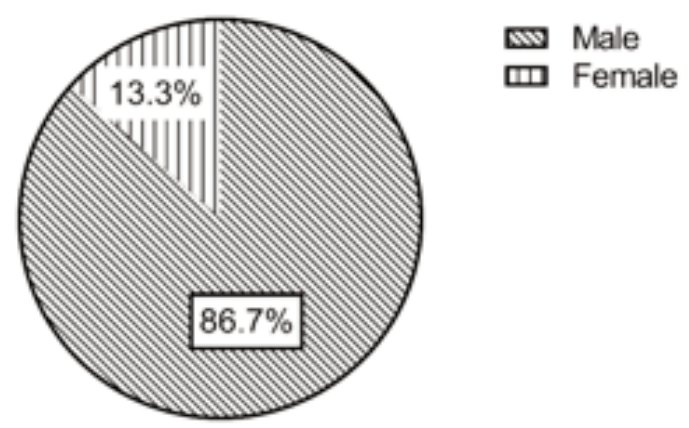

Figure 1. Pie chart of sex distribution of deaths in the ICU. Males were disproportionately involved in neurotrauma necessitating ICU admission in the University of Abuja Teaching Hospital.

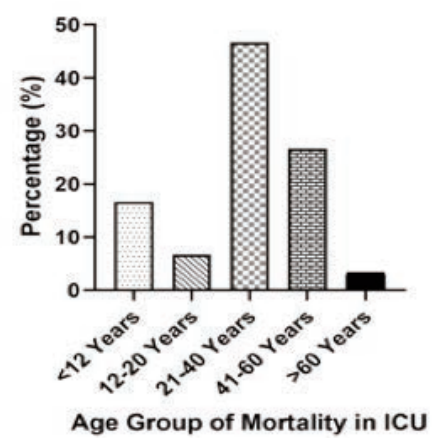

Figure 2. Bar chart of age distribution of deaths in the ICU. There was a proportionate increased mortality among the age ranges commonly involved in neurotrauma in the University of Abuja Teaching. 


\section{Use of mannitol}

All 8 patients who had mannitol administration died, given the result in Table 1. It is debatable if the death can be attributable to mannitol use or other confounding factors. Again, all the patients who had mannitol also suffered severe head injury, and so had poor prognosis. Mannitol may have a detrimental effect on mortality when compared to hypertonic saline. ${ }^{13}$ Only 3 of these patients had a cranial CT scan prior to use of mannitol, signifying that the secondary role of mannitol in increased brain swelling in the presence of active intracranial haematoma could not have been excluded. Our practice is to use mannitol after adequate resuscitation and an immediate admission cranial CT scan. In this way we avoid the detrimental effects of mannitol use including acute renal failure and instances of iatrogenic secondary brain injury. Unexpectedly, pre-admission into our hospital administration of mannitol was found in only one patient. We expected more patients with uninformed use of mannitol from peripheral health centres.

\section{Post-operative care}

Of the 10 patients who had cranial CT scan, as seen from Table 1,7 had cranial surgical procedures. While they all presented late ( $>24$ hours), they had GCS $<8$ and showed no remarkable recovery following the procedures. One of the patients with compound depressed skull fracture died of postoperative meningitis. Another with splenic rupture also died, had splenectomy; presence of extracranial injuries was a risk factor for mortality. ${ }^{10}$

\section{Length of ICU stay}

All those who died in the ICU died within one week of their admission into the ICU, as noted in Table 1. The natural course of cerebral edema occurring with head injury reaches a peak from the $5^{\text {th }}$ to the $7^{\text {th }}$ day. The deaths were increasing as the number of days increased, up to the $6^{\text {th }}$ day, contrary to the study by Tobi et al. ${ }^{14}$ which found that longer ICU-stay correlated with more patient improvement, was similar to the finding from the Korean NeuroTrauma Data Bank Investigation Study ${ }^{15}$ with $56 \%$ of head injury deaths occurring within the $1^{\text {st }}$ week. But the setting of the ICU should mitigate this. However, follow up brain CT scan were not performed. Cerebral edema can be recalcitrant to treatment occasionally. ${ }^{16}$

\section{Endotracheal intubation}

Looking further into Table 1, $12(52.6 \%)$ of the patients died while still having the indication for mechanical ventilation institution. But 4 had failed attempt at weaning off ventilatory support and died while still on the ventilator. This indicated that the majority of head injured patients in the ICU were yet to have the pathophysiological processes associated with the injury brought under control. The utility of invasive investigations like blood gas analysis, brain-specific neuromonitoring devices like intracranial pressure monitors cannot be overemphasized at such periods. But these were not available. A neurointensivist expertise was also lacking.

\section{Overall mortality}

The ICU head injury mortality among neurosurgery patients was $63.3 \%$. While Tobi et al., ${ }^{14}$ found a head injury mortality rate in ICU of $52.2 \%$, Opondo et al., ${ }^{17}$ from Kenya, East Africa, reported a mortality of $54 \%$. The mortality figure obtained was high. Achieving early ICU adherence to management guidelines is said to aid in the reduction of mortality. ${ }^{18,19}$

\section{Conclusions}

The management of head injury could be very challenging to a young and budding neurocritical unit. Head injury remain a leading cause of death among the young and productive age bracket of developing nations. The early data suggest an unusually high mortality among neurosurgical patients managed in the ICU. We recommend that while efforts are made to give the ICU its pride of place in any hospital, equipping the ICU to an optimum level including provision of brain-specific monitoring facilities should be the goal. Raising and training of neurocritical care workforce is

Table 1. Characteristics of mortality in the ICU during the study period.

\begin{tabular}{|c|c|}
\hline Characteristics & n $(\%)$ \\
\hline $\begin{array}{l}\text { Time of Presentation } \\
<1 \text { hour } \\
1-4 \text { hours } \\
4-24 \text { hours } \\
24-48 \text { hours } \\
>48 \text { hours }\end{array}$ & $\begin{array}{l}2(10.5) \\
3(15.7) \\
6(31.6) \\
7(36.8) \\
1(5.4)\end{array}$ \\
\hline $\begin{array}{l}\text { Indication for admission } \\
\text { Head injury } \\
\text { Moderate } \\
\text { Severe }\end{array}$ & $\begin{array}{c}1(5.3) \\
16(84.2)\end{array}$ \\
\hline Cervical spinal cord injury & $1(5.3)$ \\
\hline $\begin{array}{l}\text { Brain tumours - advanced } \\
\text { parotid tumour with brainstem involvement }\end{array}$ & $1(5.3)$ \\
\hline $\begin{array}{l}\text { GCS (trauma) } \\
3-8 \\
9-12 \\
13-15 \text { (Cervical spinal cord injury) } \\
\text { GCS of advanced Parotid tumour, } 4\end{array}$ & $\begin{array}{c}16(84.2) \\
1(5.3) \\
1(5.3) \\
1(5.3)\end{array}$ \\
\hline $\begin{array}{l}\text { Polytrauma } \\
\text { Yes }\end{array}$ & $5(23.3)$ \\
\hline $\begin{array}{l}\text { Mannitol } \\
\text { Yes } \\
\text { CT (those who had mannitol prior to CT scan) } \\
\text { Pre-hospital administration } \\
\text { In-hospital administration }\end{array}$ & $\begin{array}{l}8(42.1) \\
3(15.8) \\
1(5.3) \\
7(36.8)\end{array}$ \\
\hline $\begin{array}{l}\text { Post-operative } \\
\text { Elevation of depressed skull fracture } \\
\text { Extradural haematoma evacuation } \\
\text { Post-Ventriculoperitoneal shunt insertion } \\
\text { Had splenectomy }\end{array}$ & $\begin{array}{l}4(21.1) \\
3(15.8) \\
1(5.3) \\
1(5.3)\end{array}$ \\
\hline $\begin{array}{l}\text { Time to presentation to ICU (days) } \\
1 \\
2 \\
3 \\
10 \\
16\end{array}$ & $\begin{array}{l}10(52.6) \\
5(26.3) \\
2(10.5) \\
1(5.3) \\
1(5.3)\end{array}$ \\
\hline $\begin{array}{l}\text { Length of ICU Stay (days) } \\
2 \\
3 \\
4 \\
5 \\
6\end{array}$ & $\begin{array}{l}2(10.5) \\
3(15.8) \\
3(15.8) \\
5(26.3) \\
6(31.6)\end{array}$ \\
\hline $\begin{array}{l}\text { Endotracheal intubation } \\
\text { Intubated } \\
\text { Extubated } \\
\text { Failed Extubation } \\
\text { Tracheostomy }\end{array}$ & $\begin{array}{r}12(63.2) \\
5(26.3) \\
4(21.1) \\
2(10.5)\end{array}$ \\
\hline Overall mortality & $19(63.3)$ \\
\hline
\end{tabular}


another area requiring an urgent attention, towards the planning and implementation of locally agreed protocols, based on the available published evidence, which will facilitate the use of best practice and results in improved patient care. The role of health insurance policy to cater for most trauma-incident patients, is again at the fore for an effective trauma system. Though the prognosis appeared grave, instituting a well-designed protocol will achieve improved outcome.

\section{Limitations}

There were some limitations. The study was a retrospective, using death as its end point, and so neurologic outcome was not studied, which is a good prognosticator in head injury. The failure to have a cranial CT scan performed in all patients for adequate pathophysiological understanding of the injury and institution of necessary intervention is another drawback of the study, which was as a result of non-availability and lack of funds then.

\section{References}

1. Cohen A, Bodenham A, Webster N. A review of 2000 consecutive ICU admissions. Anaesthesia 1993;48:106-10.

2. Dobb GJ. Pediatric Intensive Care (editorial) Int. Care World 1993;10:165.

3. Arunodaya GR. Infections in neurology and neurosurgery in Intensive Care Units. Neurol India 2001;49:551-9.

4. Tweedie I. Neuro-critical care versus general critical care for neurological injury: Beneficial evidence. J Neuroanaesthesiol Crit Care 2016;3:62-5.

5. Suarez JI, Zaidat OO, Suri MF, et al. Length of stay and mortality in neurocritically ill patients: impact of a specialized neurocritical care team. Critical Care Med 2004;32:2311-7.

6. Varelas PN, Eastwood D, Yun HJ, et al. Impact of a neurointensivist on outcomes in patients with head trauma treated in a neurosciences intensive care unit. J Neurosurg 2006; 104:7139.

7. Adudu OP, Ogunrin OA, Adudu OG. Morbidity and mortality patterns among neurological patients in the intensive care unit of a tertiary health facility. Ann Afr Med 2007;6:174-9.

8. Oommen A. Neuro Critical Care - How it makes a Difference in Neurology. J Neurol Stroke 2015;2:00045.

9. Greenberg MS. Head trauma. In: Greenberg MS, editor. Handbook of Neurosurgery. New York: Thieme; 2010. p. 850928.

10. Schreiber MA, Aoki N, Scott BG, Beck JR. Determinants of mortality in patients with severe blunt head injury. Arch Surg 2002;137:285-90.

11. Clayton TJ, Nelson RJ, Manara AR. Reduction in mortality from severe head injury following introduction of a protocol for intensive care management. Br J Anaesth 2004;93:761-7.

12. Hardman JM, Manoukian A. Pathology of head trauma. Neuroimaging Clin N Am 2002;12:175-87.

13. Wakai A, McCabe A, Roberts I, et al. Mannitol for acute traumatic brain injury. Cochrane Database Syst Rev 2013;2013:CD001049.

14. Tobi KU, Azeez AL, Agbedia SO. Outcome of traumatic brain injury in the intensive care unit: a five-year review. S Afr J Anaesth Analg 2016;22:135-9.

15. Song SY, Lee SK, Eom KS, KNTDB Investigators. Analysis of Mortality and Epidemiology in 2617 Cases of Traumatic Brain Injury: Korean Neuro-Trauma Data Bank System 2010-2014. J Korean Neurosurg Soc 2016;59:485-91.

16. Donkin JJ, Vink R. Mechanisms of cerebral edema in traumatic brain injury: therapeutic developments. Curr Opin Neurol 2010;23:293-9.

17. Opondo EA, Mwangombe NJM. Outcome of severe traumatic brain injury at a critical care unit: a review of 87 patients. Ann Afr Surg 2004;1:1-5.

18. Gupta D, Sharma D, Kannan N, et al. Guideline Adherence and Outcomes in Severe Adult Traumatic Brain Injury for the CHIRAG (Collaborative Head Injury and Guidelines) Study. World Neurosurg 2016;89:169-79.

19. Stocchetti N, Carbonara M, Citerio G, et al. Severe traumatic brain injury: targeted management in the intensive care unit. Lancet Neurol 2017;16:452-64. 